\title{
Symmetries, Isometries and Length Spectra of Closed Hyperbolic Three-Manifolds
}

\author{
Craig D. Hodgson and Jeffrey R. Weeks
}

\section{CONTENTS}

1. Introduction

2. Finding a Complete Simple Geodesic Link

3. Length Spectra

4. Examples

Acknowledgments

Electronic Availability

Bibliography
Hodgson was partially supported by the Australian Research Council.

Weeks was partially supported by the National Science Foundation grant DMS-8920161, through the Geometry Center at the University of Minnesota.

Key words and phrases. Hyperbolic three-manifold, length spectrum, symmetry, isometry
Previously known algorithms to compute the symmetry group of a cusped hyperbolic three-manifold and to test whether two cusped hyperbolic three-manifolds are isometric do not apply directly to closed manifolds. But by drilling out geodesics from closed manifolds one may compute their symmetry groups and test for isometries using the cusped manifold techniques. To do so, one must know precisely how many geodesics of a given length the closed manifold has. Here we prove the propositions needed to rigorously compute a length spectrum, with multiplicities. We also tabulate the symmetry groups of the smallest known closed hyperbolic three-manifolds.

\section{INTRODUCTION}

A canonical cell decomposition lies at the foundation of algorithms for deciding whether or not two cusped hyperbolic three-manifolds are isometric [Hildebrand and Weeks 1989] and for computing the symmetry group of a cusped hyperbolic threemanifold [Henry and Weeks 1992]. The canonical cell decomposition depends on a convex hull construction in Minkowski space [Epstein and Penner 1988; Weeks 1993; Sakuma and Weeks 1995]. That construction makes essential use of a manifold's cusps, and does not generalize directly to closed manifolds. Fortunately we can transfer questions about closed manifolds to questions about cusped manifolds by looking at the complement of a set of disjoint simple closed geodesics.

Throughout this article, $M$ will denote a hyperbolic three-manifold of finite volume. In this introduction we further assume that $M$ is closed. We do not assume it is orientable, because our results, as well as the results we quote from [Kojima 1988] and 
[Sakai 1991], apply equally well to nonorientable manifolds.

Definition. A geodesic link is a finite set of closed geodesics in $M$. A geodesic link is simple if its constituent geodesics do not intersect themselves or each other.

Proposition 1.1 [Sakai 1991]. If $M$ is a closed hyperbolic manifold and $L$ is a simple geodesic link in $M$, the complement $M \backslash L$ admits a complete hyperbolic structure of finite volume.

Definition. The complex length of a closed geodesic is $\lambda+i \theta$, where $\lambda$ is the geodesic's length and $\theta$ is its torsion. (See sidebar on page 263.) A geodesic link $L$ is complete if it has the property that, whenever $\lambda+i \theta$ is the complex length of a geodesic in $L$, all geodesics of complex length $\lambda \pm i \theta$ are in $L$.

We denote by Isom $M$ the group of isometries from $M$ to itself. We also refer to this as the symmetry group of $M$. By Mostow rigidity, Isom $M$ is naturally isomorphic to the group of homeomorphisms from $M$ to itself, up to homotopy.

Proposition 1.2 [Kojima 1988]. If $L$ is a complete simple geodesic link in a closed hyperbolic threemanifold $M$, the group Isom $M$ is naturally isomorphic to the subgroup of $\operatorname{Isom}(M \backslash L)$ consisting of symmetries that take meridians to meridians (that is, symmetries that extend to $M$ ).

Proof. This follows immediately from Lemma 5 and Proposition 6 of [Kojima 1988]. The basic idea is that a symmetry of $M$ must preserve the complete geodesic link $L$, and therefore restricts to a homeomorphism of $M \backslash L$ to itself. By Mostow rigidity, the homeomorphism is homotopic to an isometry.

This proposition lies at the heart of our method for computing the symmetry group of a closed hyperbolic three-manifold $M$, which is, in outline, the following (see Algorithm 2.4 for a fuller version):

Algorithm 1.3. Step 1. Find a complete simple geodesic link $L$ of $M$, and drill it out.
Step 2. Compute the symmetry group of $M \backslash L$ using the canonical cell decomposition mentioned above, then take the subgroup of $\operatorname{Isom}(M \backslash L)$ that sends meridians to meridians. By Proposition 1.2, this subgroup is naturally isomorphic to Isom $M$.

Unfortunately, at step 1 we have no proof that $M$ even contains a complete simple geodesic link, let alone an algorithm guaranteed to compute one. We do, however, have a heuristic that works very well in practice. The heuristic, and the underlying mathematics, are the main topic of this article.

Step 2 is more straightforward: the canonical cell decomposition mentioned at the beginning of this section is guaranteed to exist, and the algorithm that computes it will either succeed or run forever (so far it has always succeeded quickly, and indeed one may be able to construct a statistical argument that it must eventually succeed). Given the canonical decomposition, it is straightfoward to compute $\operatorname{Isom}(M \backslash L)$, and to check which of its elements preserve meridians.

A similar technique allows us to test whether two closed hyperbolic three-manifolds $M$ and $M^{\prime}$ are isometric: we drill corresponding complete geodesic links out of both $M$ and $M^{\prime}$, then use canonical decompositions to check whether $M \backslash L$ and $M^{\prime} \backslash L^{\prime}$ are isometric by a meridian-preserving isometry.

The computer program SnapPea [Weeks 1995] implements Algorithm 1.3, as well as other algorithms for creating and studying hyperbolic threemanifolds. The logical status of these algorithms varies: some are rigorous and guaranteed to produce correct results in a finite number of steps; others, like the one just described, are heuristics that work well in practice but could in principle fail; still others, like the length spectrum algorithm described in Section 3 below, are rigorous in the strict mathematical sense, but in practice are subject to the limitations of fixed-precision floating point computations.

The rest of this paper is structured as follows. Section 2 discusses our heuristics for finding a complete simple geodesic link, first presenting the basic 


\section{ISOMETRIES OF $\mathbb{H}^{3}$}

The classification of orientation-preserving isometries of hyperbolic three-space $\mathbb{H}^{3}$, and their relation to elements of $\operatorname{PSL}(2, \mathbb{C})$, is very well-known (see [Beardon 1983, Section 4.3], for example). The classification of orientationreversing isometries is less familiar, so we outline it here for convenience, showing how geometric information can be derived from algebraic data.

Recall that Minkowski space $\mathbb{E}^{3,1}$ is $\mathbb{R}^{4}$ with a quadratic form $v$ of signature $(3,1)$, which we take as $v(x, y)=$ $-u_{0} v_{0}+u_{1} v_{1}+u_{2} v_{2}+u_{3} v_{3}$ for concreteness. $\mathbb{H}^{3}$ has a natural embedding in $\mathbb{E}^{3,1}$ as either of the two sheets of the hyperboloid $|v|=-1$. The isometry group $O(3,1)$ of $\mathbb{E}^{3,1}$ has a subgroup $O^{+}(3,1)$ of index two that preserves each sheet, and which can be regarded as the isometry group of $\mathbb{H}^{3}$. Further, $O^{+}(3,1)$ has two components, consisting respectively of orientation-preserving and orientation-reversing transformations.

An orientation-preserving transformation that is not the identity fixes either one or two points at infinity (isotropic vectors of $v$ up to scalar multiplication). If there is one fixed point at infinity, the transformation is parabolic. If there are two, the transformation is elliptic if it has fixed points in $\mathbb{H}^{3}$ itself and hyperbolic otherwise (we do not distinguish between screw motions and translations). By conjugation, we can reduce any orientation-preserving transformation to one of the following forms:

$$
\begin{aligned}
& \text { identity parabolic elliptic } \\
& \left(\begin{array}{llll}
1 & 0 & 0 & 0 \\
0 & 1 & 0 & 0 \\
0 & 0 & 1 & 0 \\
0 & 0 & 0 & 1
\end{array}\right) \quad\left(\begin{array}{rrrr}
\frac{3}{2} & -\frac{1}{2} & 1 & 0 \\
\frac{1}{2} & \frac{1}{2} & 1 & 0 \\
1 & -1 & 1 & 0 \\
0 & 0 & 0 & 1
\end{array}\right) \quad\left(\begin{array}{cccc}
1 & 0 & 0 & 0 \\
0 & 1 & 0 & 0 \\
0 & 0 & \cos \theta & -\sin \theta \\
0 & 0 & \sin \theta & \cos \theta
\end{array}\right) \quad\left(\begin{array}{cccc}
\cosh \lambda & \sinh \lambda & 0 & 0 \\
\sinh \lambda & \cosh \lambda & 0 & 0 \\
0 & 0 & \cos \theta & -\sin \theta \\
0 & 0 & \sin \theta & \cos \theta
\end{array}\right)
\end{aligned}
$$

Conjugacy classes of hyperbolic and elliptic orientation-preserving isometries, taken together, form a twoparameter family, parametrized by the complex number $\lambda+i \theta$ (defined modulo $2 \pi i$ ). This number is called the complex length associated with the transformation, and also the complex length of the corresponding geodesic in the quotient manifold; it equals $2 \operatorname{arccosh}\left(\frac{1}{2} \operatorname{tr}\right)$, where $\operatorname{tr}$ is the complex-valued trace of the corresponding matrix in $\operatorname{PSL}(2, \mathbb{C})$ (which is determined up to sign).

An orientation-reversing transformation $g$ always leaves some plane $P$ globally invariant. By composing with reflection in that plane, we reduce to an orientation-preserving transformation $f$ that leaves the same plane invariant. Therefore $f$ is elliptic, parabolic, or hyperbolic with $\theta=0$, and we assign $g$ the same type as $f$, unless $g$ is a pure reflection in a plane orthogonal to $P$ (in which case $f$ is elliptic with $\theta=180^{\circ}$ ). A pure reflection may be considered a degenerate isometry of any of the three types. Thus, by conjugation, we can reduce any orientation-reversing transformation to one of the following forms:

$$
\begin{gathered}
\text { pure reflection } \\
\left(\begin{array}{rrrr}
1 & 0 & 0 & 0 \\
0 & -1 & 0 & 0 \\
0 & 0 & 1 & 0 \\
0 & 0 & 0 & 1
\end{array}\right) \quad\left(\begin{array}{rrrr}
\frac{3}{2} & -\frac{1}{2} & 1 & 0 \\
\frac{1}{2} & \frac{1}{2} & 1 & 0 \\
1 & -1 & 1 & 0 \\
0 & 0 & 0 & -1
\end{array}\right) \quad\left(\begin{array}{rrrrr}
1 & 0 & 0 & 0 \\
0 & -1 & 0 & 0 \\
0 & 0 & \cos \theta & -\sin \theta \\
0 & 0 & \sin \theta & \cos \theta
\end{array}\right) \quad\left(\begin{array}{cccc}
\cosh \lambda & \sinh \lambda & 0 & 0 \\
\sinh \lambda & \cosh \lambda & 0 & 0 \\
0 & 0 & 1 & 0 \\
0 & 0 & 0 & -1
\end{array}\right)
\end{gathered}
$$

$\left.\begin{array}{ccc}\sinh \lambda & 0 & 0 \\ \cosh \lambda & 0 & 0 \\ 0 & 1 & 0 \\ 0 & 0 & -1\end{array}\right)$

We see that hyperbolic and elliptic orientation-reversing isometries form separate one-parameter families, parametrized up to conjugacy by the real length $\lambda$ or the rotation angle $\theta$. Here too we call $\lambda+i \theta$ the complex length associated with the transformation. These numbers are determined by the trace of the transformation's matrix in $O(3,1)$ : this can be seen by inspecting the standard forms just given and using the fact that the trace is a conjugacy invariant. Thus we can read geometric information off the matrix, as follows. An orientation-reversing transformation of trace less than 2 is an elliptic isometry of rotation angle $\theta=\arccos \left(\frac{1}{2} \operatorname{tr}\right)$, and an orientation-reversing transformation of trace greater than 2 is an hyperbolic isometry of translation distance $\lambda=\operatorname{arccosh}\left(\frac{1}{2} \operatorname{tr}\right)$. An orientation-reversing transformation of trace 2 may be either parabolic or a pure reflection. 
idea and then elaborating as shortcomings of that idea become obvious. Inasmuch as the heuristics have been implemented in SnapPea, we frame the discussion in terms of SnapPea's capabilities and algorithms, but the theoretical results that accompany this discussion should make it clear that the algorithms are independent of any particular implementation.

In Section 3 we present a rigorous algorithm for computing how many geodesics of a given complex length appear in the manifold, a computation that is required by the heuristic algorithm of Section 2 .

Finally, Section 4 lists the results of the application of this paper's techniques to a number of closed hyperbolic three-manifolds of small volume.

\section{FINDING A COMPLETE SIMPLE GEODESIC LINK}

The only curves that SnapPea can drill out are those that embed in the dual one-skeleton of a triangulation representing a given manifold. SnapPea prepares a list of all such curves, giving the complex length of the unique geodesic in each homotopy class, and the user (who may be either a human or another computer program) says which curve he, she or it would like drilled out. To drill out a complete simple geodesic link, we ask SnapPea to successively drill out several curves of the same length. However, several pitfalls await us.

(a) A desired geodesic may not be homotopic to any simple closed curve in the dual one-skeleton, in which case we can't drill it out.

(b) A curve in the dual one-skeleton may be homotopic, but not isotopic, to a desired geodesic. In this case SnapPea will drill out a curve in the wrong isotopy class.

(c) Even if we avoid (a) and (b) and successfully drill out a complete simple geodesic link, we won't know a priori that we've done so.

We can avoid pitfall (c) if we know in advance how many curves of a given complex length our manifold contains. In Section 3 we will present results that allow us to compute a length spectrum with multiplicities, so SnapPea is able to successfully deal with (c).

As for pitfall (b), when we drill out a curve in the wrong isotopy class, we still obtain some useful information. If $L^{\prime}$ is a link that is homotopic but not isotopic to the desired geodesic link $L$, and if $M \backslash L^{\prime}$ is hyperbolic, the group $\operatorname{Isom}\left(M \backslash L^{\prime}\right)$ will be a subgroup of Isom $M$. Roughly speaking, every symmetry of $M \backslash L^{\prime}$ that preserves meridians on the boundary extends to a symmetry of $M$, but not every symmetry of $M$ restricts to a symmetry of $M \backslash L^{\prime}$.

Proposition 2.1. If $L^{\prime}$ is a set of nonintersecting (not necessarily geodesic) simple closed curves in $M$, and if $M \backslash L^{\prime}$ is hyperbolic, the subgroup of isometries of $M \backslash L^{\prime}$ that sends meridians to meridians injects naturally into Isom $M$.

Proof. This follows from an observation of Sakuma, quoted in the proof of [Kojima 1988, Prop. 6]. A theorem of Borel [Conner and Raymond 1972] states that if $G$ is a finite group acting effectively on a closed aspherical manifold $M$ and $\pi_{1}(M)$ has trivial center, the induced homomorphism from $G$ to Out $\pi_{1}(M)$ is injective. So let $G$ be the subgroup of $\operatorname{Isom}\left(M \backslash L^{\prime}\right)$ that sends meridians to meridians, and then apply Mostow rigidity to equate Out $\pi_{1}(M)$ with Isom $M$.

Roughly speaking, Isom $\left(M \backslash L^{\prime}\right)$ gives us a lower bound on the symmetry group Isom $M$. Our strategy is to look for an upper bound on the order of Isom $M$. If our upper bound equals the order of $\operatorname{Isom}\left(M \backslash L^{\prime}\right)$, we know that $\operatorname{Isom}\left(M \backslash L^{\prime}\right)=$ Isom $M$.

To obtain an upper bound, we have SnapPea drill out a simple closed curve $K$ from the dual one-skeleton of the given triangulation of $M$. It follows from Thurston's theory of hyperbolic Dehn filling [Thurston 1979, Ch. 4] that, if the hyperbolic structure on $M$ is the metric completion of an incomplete hyperbolic structure on $M \backslash K$ triangulated by positively oriented ideal tetrahedra, the curve $K$ must be isotopic to a geodesic. In 
this case, Proposition 2.2 below lets us compute the subgroup of Isom $M$ that preserves the unique geodesic isotopic to $K$, and Proposition 2.3 then gives us an upper bound on $\mid$ Isom $M \mid$.

Let $\operatorname{Isom}(M, L)$ be the subgroup of Isom $M$ that preserves a geodesic link $L$.

Proposition 2.2 [Kojima 1988]. Let $M$ be a closed hyperbolic three-manifold. If $L$ is a (not necessarily complete) simple geodesic link in $M$, the group $\operatorname{Isom}(M, L)$ is naturally isomorphic to the subgroup of $\operatorname{Isom}(M \backslash L)$ that takes meridians to meridians.

Proof. Just like Proposition 1.2, this follows from [Kojima 1988, Lemma 5 and Prop. 6].

Proposition 2.3. Let $K$ be a simple geodesic knot in $M$ with complex length $\lambda+i \theta$, and let $n$ be the number of closed geodesics of $M$ with complex length $\lambda \pm i \theta$. Then $|\operatorname{Isom} M| \leq n|\operatorname{Isom}(M, K)|$.

Proof. Let $S$ be the set of closed geodesics with complex length $\lambda \pm i \theta$. Symmetries of $M$ preserve length and absolute value of torsion for geodesics, so the group Isom $M$ acts on $S$. The stabilizer of $K$ is $\operatorname{Isom}(M, K)$, so the orbit of $K$ has order equal to $|\operatorname{Isom} M| /|\operatorname{Isom}(M, K)| \leq|S|=n$.

In summary, here is our procedure for computing symmetry groups of closed hyperbolic manifolds, as implemented in SnapPea:

\section{Algorithm 2.4 (Computing symmetry groups).}

Input: A closed hyperbolic manifold $M$.

Output: The symmetry group of $M$, or failure.

- Compute the length spectrum of $M$ up to some maximum length $\lambda$ (see Section 3; for low-volume manifolds $\lambda=1.5$ works well).

- For each complex length in the length spectrum:

- Try to drill out a single curve $K$ of that complex length.

- If $M$ can be recovered as a hyperbolic Dehn filling on $M \backslash K$ with all positively oriented tetrahedra, apply Proposition 2.3 to obtain an upper bound on $\mid$ Isom $M \mid$.

- Try to drill out the complete geodesic link $L$ consisting of all curves of the given length and absolute value of torsion. If this is possible, use Proposition 2.1 to obtain a lower bound on the order of Isom $M$.

- If the best lower bound equals the least upper bound, the best lower bound is indeed $|\operatorname{Isom} M|$. - Else, return failure.

Note that the algorithm may fail to drill out the desired curves at any step (see pitfall (a) on the preceding page). Even if it does drill out the desired curves, the presence of negatively oriented tetrahedra may prevent us from establishing an upper bound on the order of $|\operatorname{Isom} M|$. Nevertheless, the algorithm works well in practice: it successfully computes the symmetry group of all but five of the 11,031 distinct low-volume closed hyperbolic threemanifolds studied in [Hodgson and Weeks]. The authors have computed the symmetry groups of the five exceptional cases using ad hoc techniques.

Question. Do all closed hyperbolic three-manifolds contain a complete simple geodesic link?

\section{LENGTH SPECTRA}

As explained in Section 1, to drill out a complete simple geodesic link we need to know how many geodesics of a given complex length appear in $M$. In other words, we need a length spectrum with multiplicities. The remainder of this section explains SnapPea's algorithm for rigorously computing such a length spectrum.

Notation. Throughout this section $M$ represents a hyperbolic three-dimensional manifold or orbifold of finite volume, and $x \in \mathbb{H}^{3}$ is an arbitrary basepoint in the universal cover $\mathbb{H}^{3}$ of $M$. If $M$ is an orbifold, we assume that $x$ does not lie in the preimage of the singular set (that is, $x$ is not a fixed point of any covering transformation).

The key idea is to begin with a Dirichlet domain for $M$. We recall the definition. For each covering transformation $g$ of $M$, let $H_{g}$ be the half-space consisting of points at least as close to $x$ as to $g x$ :

$$
H_{g}=\left\{p \in \mathbb{H}^{3} \mid d(p, x) \leq d(p, g x)\right\} .
$$


The Dirichlet domain $D$ of $M$ (with basepoint $x$ ) is the intersection of the $H_{g}$ for all covering transformations $g$. Because $M$ is geometrically finite, $D$ is a finite-sided polyhedron. If $M$ is a noncompact, some of the vertices of $D$ will be ideal points on the sphere at infinity.

Remark. A choice of basepoint $x$ such that the distance $d(x, g x)$ from $x$ to its nearest translate is maximal typically leads to a more symmetrical Dirichlet domain, and also to a smaller spine radius (discussed below). However, all results in this section are valid for any choice of basepoint.

For the present purposes we assume given a Dirichlet domain. SnapPea computes Dirichlet domains using an algorithm that usually succeeds, but can fail for large manifolds because of the rapidly accumulating round-off errors inherent in matrix multiplication in $O(3,1)$. (For a brief explanation of this algorithm, see [Hodgson and Weeks]. A more detailed exposition may appear later; meanwhile one may consult the extensive documentation in the files Dirichlet.c, Dirichlet.h, Dirichlet basepoint.c and Dirichlet_construction.c in [Weeks 1995].)

Let $D$ be a Dirichlet domain for $M$ with basepoint $x$. A typical translate of $D$ will be denoted $g D$, where $g$ is an isometry in the group of covering transformations.

We'll tile an approximate ball in $\mathbb{H}^{3}$ with translates $g D$ of $D$. Each primitive hyperbolic isometry $g$ corresponds to a translation along a geodesic, perhaps followed by a rotation or reflection fixing the geodesic pointwise. (See the sidebar on page 263.)

We'd like to find all translates $g D$ of $D$ that move the basepoint $x$ a distance less than some given distance $s$, that is, all $g D$ such that $d(x, g x)<$ $s$. The simplest algorithm is to start with $D$ and recursively attach its neighbors, stopping the recursion when we reach translates $g D$ that move the basepoint a distance greater than $s$-that is, when $d(x, g x)>s$. For an arbitrary fundamental domain (not necessarily a Dirichlet domain) with an arbitrary basepoint, this algorithm might fail: there could be a translate moving the basepoint a distance less than $s$, all of whose neighbors move the basepoint a distance greater than $s$. The simple recursive algorithm would not find such a translate. Fortunately this cannot occur for a Dirichlet domain.

Proposition 3.1. Let $D$ be a Dirichlet domain with basepoint $x$, and let $g D$ be a translate of $D$ such that $d(x, h x) \geq d(x, g x)$ for all neighbors $h D$ of $g D$. Then $g$ is the identity.

Proof. For each neighbor $h D$ of $g D$, the inequality $d(x, h x) \geq d(x, g x)$ implies that $x$ lies in the halfspace $H_{h}$ consisting of points closer to $g x$ than $h x$. But $g D$ is the intersection of all such $H_{h}$, so $x$ must lie in $g D$. Our assumption that no covering transformation fixes $x$ implies that no translate $g D$ contains $x$, except for $D$ itself. Therefore $g D=D$, and $g$ is the identity.

If we want to find all geodesics of length at most $\lambda$, to what radius $s$ must we tile $\mathbb{H}^{3}$ ? The answer to this question depends not only on $\lambda$, but also on the size of the Dirichlet domain. The relevant measure of size is the "spine radius", which we are about to define. Proposition 3.3 provides a practical means of computing the spine radius. Proposition 3.4 then expresses the required tiling radius $s$ in terms of the spine radius $r$ and the cutoff length $\lambda$.

To introduce the spine radius, we observe that the Dirichlet domain $D$, with faces identified, defines a cell decomposition $K$ for $M$. Let $K^{\prime}$ be a cell decomposition of $M$ dual to $K$, including the "three-cells" dual to any ideal vertices of $K$. The two-skeleton of $K^{\prime}$ is a spine dual to the Dirichlet domain.

Proposition 3.2. A spine dual to the Dirichlet domain must intersect every closed geodesic of $M$.

Proof. Let $K$ and $K^{\prime}$ be as above. The three-cells of $K^{\prime}$ are in one-to-one correspondence with the vertices of $K$. If $M$ is a manifold, each three-cell 
of $K^{\prime}$ will be either a topological ball (if the corresponding vertex of $K$ is finite) or a topological torus or Klein bottle cross a half line (if the corresponding vertex of $K$ is ideal). If $M$ is an orbifold, a three-cell dual to a finite vertex may be a cone on any spherical two-orbifold, and a three-cell dual to an infinite vertex may be any Euclidean twoorbifold cross a half line. We know the three-cells must take this form because the singular set (if any) must be contained in the Dirichlet domain's boundary; this follows from our assumption that the basepoint $x$ does not lie in the preimage of the singular set. It follows that no geodesic may lie entirely in a single three-cell of $K^{\prime}$, since it would then be contractible (or perhaps boundary parallel if $M$ has cusps). Therefore every geodesic must intersect the two-skeleton.

Definition. The radius of a spine dual to a Dirichlet domain is the maximum distance from a point in the spine to the basepoint. The Dirichlet domain's spine radius is the infimum of the radii of all spines dual to the domain.

The following definition is local to the next proposition. The maximin edge distance of a Dirichlet domain is the maximum over all the domain's edges of the minimum distance from the edge to the basepoint $x$.

Proposition 3.3. The spine radius is the maximin edge distance.

Proof. Any spine dual to the Dirichlet domain must intersect every edge, so the spine radius is greater than or equal to the maximin edge distance. It remains to show that for any $\varepsilon$ greater than zero, we can construct a spine whose radius is within $\varepsilon$ of the maximin edge distance. Carry out the following construction in the Dirichlet domain $D$ (not the space $M$ obtained by identifying faces), replacing $\varepsilon$ by a smaller value if necessary.

Step 1. On each edge, mark the point closest to the basepoint. If that point is at an endpoint, displace it a distance $\varepsilon$ into the interior of the edge. The edge identifications respect the marked points, and the marked points all lie within the maximin edge distance plus $\varepsilon$ of the basepoint.

Step 2. On each face, mark the point closest to the basepoint. If that point is on the boundary, displace it a distance $\varepsilon$ into the interior of the face (towards the center, say). The face identifications respect the marked points, and the marked points all lie within the maximin edge distance plus $\varepsilon$ of the basepoint.

Step 3. Draw lines from the marked point in the interior of each face to the marked points on the incident edges. The face identifications respect the lines, and the lines all lie within the maximin edge distance plus $\varepsilon$ of the basepoint.

Step 4. Cone the complex created in steps 1-3 to the basepoint. This gives a spine dual to the $\mathrm{Di}$ richlet domain and lying within the maximin edge distance plus $\varepsilon$ of the basepoint.

Note that the spine radius is finite even for $\mathrm{Di}$ richlet domains of cusped manifolds, whose radius is infinite. As already mentioned, the techniques of this section work to compute length spectra for cusped manifolds as well as closed ones.

Remark. In practice, in order to improve performance, SnapPea removes certain two-cells from the spine to reduce its radius without compromising the fact that every geodesic must intersect it. This modification does not affect the basic theory.

For the remainder of this section, we denote by $r$ the spine radius of the Dirichlet domain $D$.

Proposition 3.4. To find all closed geodesics of length at most $\lambda$, it suffices to find all translates $g D$ such that $d(x, g x) \leq \lambda+2 r$.

Proof. For each closed geodesic $\gamma$ of length at most $\lambda$, we must find a covering transformation $g$ whose axis is a preimage of the geodesic $\gamma$ and which satisfies $d(x, g x)<\lambda+2 r$.

Choose $\varepsilon>0$, and construct a spine $S$ dual to $D$ with radius at most $r+\varepsilon$. (See Figure 1.) By 


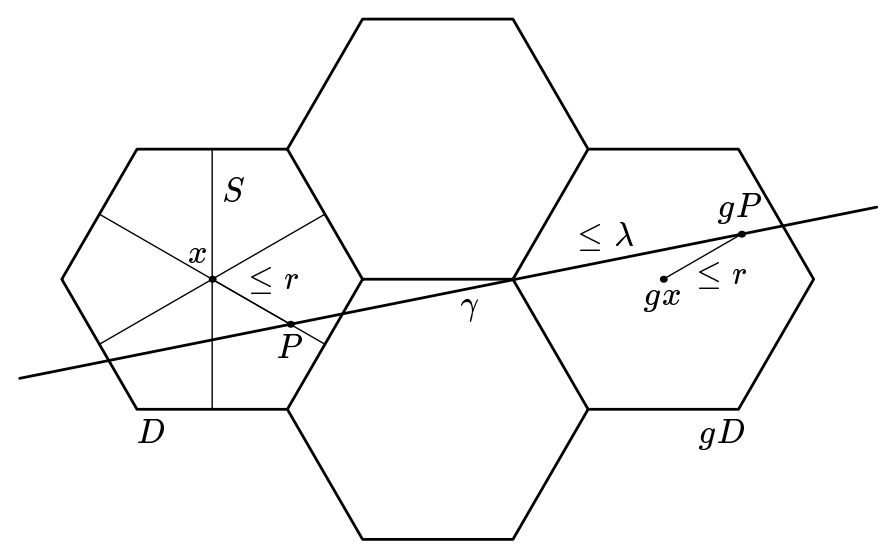

FIGURE 1. The distance from $x$ to $g x$ is at most $r+\lambda+r$, where $\lambda$ is the translation length of $g$.

Proposition 3.2, a lift of the geodesic $\gamma$ must intersect $S$ at some point $P$. Let $g$ be the covering transformation corresponding to this lift of $\gamma$. Then $d(P, g P)=$ length $g$, and

$$
\begin{aligned}
d(x, g x) & \leq d(x, P)+d(P, g P)+d(g P, g x) \\
& \leq(r+\varepsilon)+\lambda+(r+\varepsilon)=\lambda+2 r+2 \varepsilon .
\end{aligned}
$$

Let $\varepsilon$ go to zero to obtain $d(x, g x) \leq \lambda+2 r$.

With a little extra care, we can improve on the estimate of $\lambda+2 r$.

Proposition 3.5. To find all closed geodesics of length at most $\lambda$, it suffices to find all translates $g D$ such that $d(x, g x) \leq 2 \cosh ^{-1}\left(\cosh r \cosh \left(\frac{1}{2} \lambda\right)\right)$.

Proof. Same as that of Proposition 3.4, except that instead of using a point $P$ where $\gamma$ intersects the spine, we use a point $Q$ at which the distance from $\gamma$ to the basepoint $x$ is a minimum (Figure 2). For all choices of $\varepsilon$ and $S$ we have $d(x, Q) \leq d(x, P) \leq$ $r+\varepsilon$, so $d(x, Q) \leq r$. The advantage of using $Q$ instead of $P$ is that the segment from $x$ to $Q$ is orthogonal to the geodesic $\gamma$. If we let $M$ be the midpoint of the segment from $Q$ to $g Q$ we can use hyperbolic trigonometry to bound the distance from $x$ to $M$ as $d(x, M) \leq \cosh ^{-1}\left(\cosh r \cosh \left(\frac{1}{2} \lambda\right)\right)$. The distance from $x$ to $\gamma x$ will be at most twice that.

Remark. Empirical tests show that for small manifolds the length spectrum computation runs about

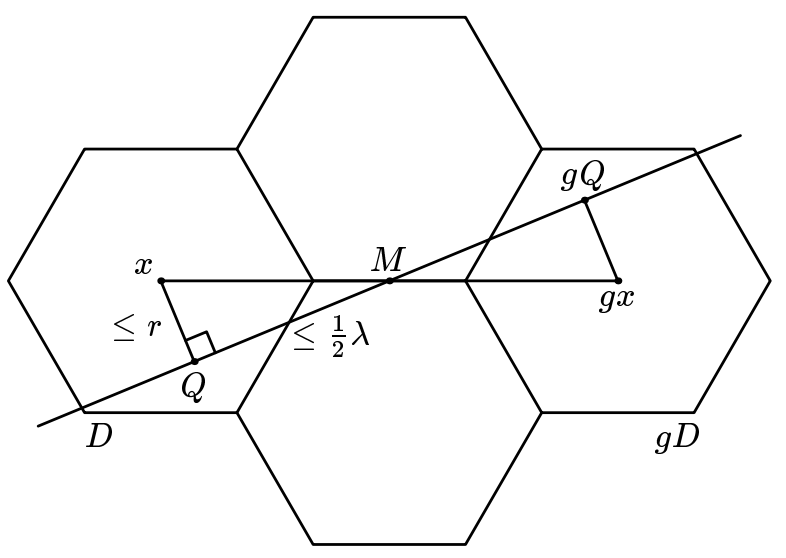

FIGURE 2. The distance from $x$ to $g x$ is at most $2 \cosh ^{-1}\left(\cosh r \cosh \left(\frac{1}{2} \lambda\right)\right)$.

five times faster with the bound of Proposition 3.5 than with that of Proposition 3.4. Theoretical estimates predict that, as $r$ and $\lambda$ go to infinity, the run-time difference approaches a factor of 16 .

Proposition 3.5 tells us how to find group elements corresponding to all geodesics of length at most $\lambda$. But different groups elements may be conjugate to one another and correspond to the same geodesic. To find a length spectrum with correct multiplicities, we must find precisely one group element in each conjugacy class. The following algorithm does that; in its statement, the big list is the complete set of group elements $g$ satisfying $d(x, g x) \leq 2 \cosh ^{-1}\left(\cosh r \cosh \left(\frac{1}{2} \lambda\right)\right)$, and the small list is a subset of the big list that gets whittled down until precisely one element of each conjugacy class remains.

\section{Algorithm 3.6 (Removing duplicates).}

(Initialize) Set the small list equal to the big list.

Step 1. Remove group elements whose corresponding complex lengths have real part zero. If $M$ is a manifold these elements will be parabolic, but if $M$ is an orbifold elliptics may also occur. Also remove group elements of real length greater than $\lambda$.

Step 2. Remove group elements whose axes don't pass within a distance $r$ of the basepoint. (Every geodesic must intersect a spine of radius $r+\varepsilon$, for all $\varepsilon>0$, so we are sure to retain at least one element in every conjugacy class.) 
Step 3. Remove group elements that are powers of others. We aren't interested in a "geodesic" that is just a shorter geodesic traversed twice.

Step 4. Check which of the elements remaining on the small list are conjugate to one another. Proposition 3.7 below ensures that if two elements of the small list are conjugate to each other, the conjugacy is realized by an element from the big list. That is, if two elements $g_{1}$ and $g_{2}$ on the small list are conjugate, there exists an element $h$ on the big list such that $g_{1}=h g_{2} h^{-1}$. Keep precisely one element corresponding to each geodesic, and discard its conjugates, its inverse, and the conjugates of its inverse. (In a manifold an element and its inverse will be in different conjugacy classes, but in an orbifold they may be in the same conjugacy class, in which case the geodesic is topologically a mirrored interval rather than a circle.)

The resulting small list yields a length spectrum with correct multiplicities.

Proposition 3.7. If $g_{1}$ and $g_{2}$ are conjugate group elements corresponding to a geodesic of length at most $\lambda$, and both their axes pass within a distance $r$ of the basepoint, then there is a group element $h$ such that $g_{1}=h g_{2} h^{-1}$ and

$$
d(x, h x) \leq 2 \cosh ^{-1}\left(\cosh r \cosh \left(\frac{1}{4} \lambda\right)\right) .
$$

Proof. Let $A_{i}$ be the axis of $g_{i}$, and $Q_{i}$ be the point on $A_{i}$ closest to the basepoint $x$. (See Figure 3.) There are infinitely many covering transformations taking $A_{1}$ to $A_{2}$; let $h$ be one that minimizes the distance from $h Q_{1}$ to $Q_{2}$. Because the length of the underlying geodesic is at most $\lambda$, the distance from $h Q_{1}$ to $Q_{2}$ is at most $\frac{1}{2} \lambda$ (if instead $\frac{1}{2} \lambda<d\left(h Q_{1}, Q_{2}\right)<\lambda$ we got the wrong $h$-we need to consider an $h$ that takes $Q_{1}$ to a point on the other side of $Q_{2}$ ). Now imitate the proof of Proposition 3.5 to get the desired bound for $d(x, h x)$.

The bound on $d(x, h x)$ in Proposition 3.7 is less than the bound on $d(x, g x)$ in Proposition 3.5. This implies that, as claimed, the big list contains all

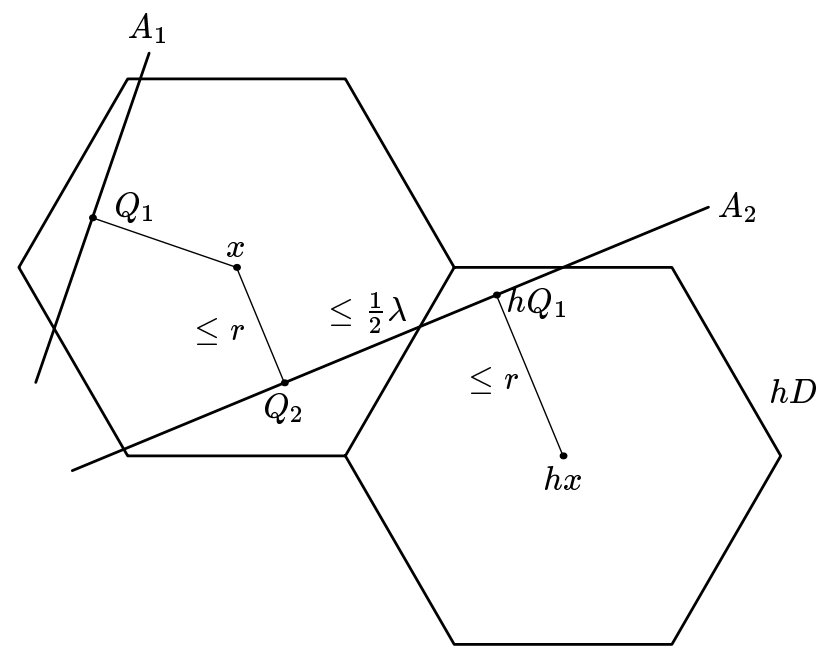

FIGURE 3. The distance from $x$ to $h x$ is at most $2 \cosh ^{-1}\left(\cosh r \cosh \left(\frac{1}{4} \lambda\right)\right)$.

the conjugating matrices we could possibly need in Step 4 of the above algorithm.

Remark. The methods of this section may also be applied to compute the based ortholength spectrum associated to a fixed geodesic.

\section{EXAMPLES}

Knowing the lengths and multiplicities of the shortest geodesics, we applied the technique outlined in Section 1 to compute the symmetry groups of the smallest known closed hyperbolic three-manifolds, which are part of a census of closed hyperbolic three-manifolds described in [Hodgson and Weeks] (see also the section on Electronic Availability at the end of the text).

Table 1 gives the results for the ten smallest manifolds. Table 2 provides examples of closed nonorientable hyperbolic three-manifolds, and Table 3 lists length spectra for some sample orbifolds (although the results of Section 3 apply to orbifolds as well as manifolds, we cannot in general compute the symmetry group of an orbifold).

On a Macintosh SE/30, each length spectrum computation took about 30 seconds. The other computations were almost instantaneous. 


\begin{tabular}{|c|c|c|c|c|c|}
\hline $\begin{array}{c}1 \\
-3,2\end{array}$ & $\begin{array}{l}\text { vol }=0.94270736 \\
\mathrm{CS}=0.06004307 \\
H_{1}=\mathbb{Z}_{5}+\mathbb{Z}_{5} \\
\text { Isom }=D_{6}\end{array}$ & $\begin{array}{l}(\times 3) \\
(\times 3)\end{array}$ & $\begin{array}{l}0.58460369+2.49537046 i \\
0.79413466-2.30485682 i\end{array}$ & $(\times 3)$ & $1.28985116+2.48392462 i$ \\
\hline 2 & $\begin{array}{l}\text { vol }=0.98136883 \\
\mathrm{CS}=0.07703818 \\
H_{1}=\mathbb{Z}_{5} \\
\text { Isom }=D_{2}\end{array}$ & $\begin{array}{l}(\times 1) \\
(\times 1) \\
(\times 2) \\
(\times 2)\end{array}$ & $\begin{array}{l}0.57808244+2.13243064 i \\
0.72156837-1.15121299 i \\
0.88944300+2.94185905 i \\
0.99832519-2.92101779 i\end{array}$ & $\begin{array}{l}(\times 1) \\
(\times 2) \\
(\times 1)\end{array}$ & $\begin{array}{l}1.04031513+0.98237189 i \\
1.79380084-1.55687105 i \\
1.82227970-2.41353903 i\end{array}$ \\
\hline 3 & $\begin{array}{l}\text { vol }=1.01494161 \\
\mathrm{CS}=0 \\
H_{1}=\mathbb{Z}_{3}+\mathbb{Z}_{6} \\
\text { Isom }=S_{16}\end{array}$ & $\begin{array}{l}(\times 2) \\
(\times 2) \\
(\times 2) \\
(\times 2)\end{array}$ & $\begin{array}{l}0.83144295-1.94553076 i \\
0.83144295+1.94553076 i \\
0.86255463-2.68067319 i * \\
0.86255463+2.68067319 i *\end{array}$ & $\begin{array}{l}(\times 1) \\
(\times 2) \\
(\times 2)\end{array}$ & $\begin{array}{l}1.31695790+3.14159265 i \\
1.99165239-1.29953257 i \\
1.99165239+1.29953257 i\end{array}$ \\
\hline 4 & $\begin{array}{l}\text { vol }=1.26370924 \\
\mathrm{CS}=0.11414066 \\
H_{1}=\mathbb{Z}_{5}+\mathbb{Z}_{5} \\
\text { Isom }=D_{4}\end{array}$ & $\begin{array}{l}(\times 2) \\
(\times 2) \\
(\times 4)\end{array}$ & $\begin{array}{l}0.57507858+1.74532235 i \\
0.66683593-2.82503375 i \\
1.16858110+2.63105912 i\end{array} *$ & $\begin{array}{l}(\times 2) \\
(\times 2)\end{array}$ & $\begin{array}{l}1.80421804-1.52234769 i \\
1.81598680+2.23627048 i\end{array}$ \\
\hline 5 & $\begin{array}{l}\text { vol }=1.28448530 \\
\mathrm{CS}=0.06793167 \\
H_{1}=\mathbb{Z}_{6} \\
\text { Isom }=D_{2}\end{array}$ & $\begin{array}{l}(\times 1) \\
(\times 1) \\
(\times 2) \\
(\times 1) \\
(\times 2)\end{array}$ & $\begin{array}{l}0.48031180-0.93377666 i \\
0.75757225+2.06174612 i \\
1.14870610+2.85490514 i \\
1.26692640+1.02808709 i \\
1.36531695-2.95884090 i\end{array}$ & $\begin{array}{l}(\times 1) \\
(\times 1) \\
(\times 1) \\
(\times 1)\end{array}$ & $\begin{array}{l}1.44093541-2.80132997 i \\
1.51514450-2.15969307 i \\
1.56188878+1.21107398 i \\
1.90657583-1.45364261 i\end{array}$ \\
\hline 6 & $\begin{array}{l}\text { vol }=1.39850888 \\
\mathrm{CS}=-0.24660725 \\
H_{1}=\text { trivial } \\
\text { Isom }=D_{2}\end{array}$ & $\begin{array}{l}(\times 1) \\
(\times 1) \\
(\times 1) \\
(\times 2)\end{array}$ & $\begin{array}{l}0.36613070+3.11358994 i \\
0.90689558+1.35222273 i \\
1.07293593-1.29048759 i \\
1.43906664+1.82310235 i\end{array}$ & $\begin{array}{l}(\times 2) \\
(\times 1) \\
(\times 1)\end{array}$ & $\begin{array}{l}1.69233833-1.75385127 i \\
1.77974117+0.58862018 i \\
1.90415991-0.62376635 i\end{array}$ \\
\hline 7 & $\begin{array}{l}\text { vol }=1.41406104 \\
\mathrm{CS}=-0.07660207 \\
H_{1}=\mathbb{Z}_{6} \\
\text { Isom }=D_{2}\end{array}$ & $\begin{array}{l}(\times 1) \\
(\times 1) \\
(\times 1) \\
(\times 1)\end{array}$ & $\begin{array}{l}0.79413466-2.30485682 i \\
0.84359872+1.40771544 i \\
0.85397176-0.88606928 i \\
1.08957507+1.70114752 i\end{array} *$ & $\begin{array}{l}(\times 1) \\
(\times 2) \\
(\times 2) \\
(\times 1)\end{array}$ & $\begin{array}{l}1.18953767-2.75480329 i \\
1.23287808-2.96153066 i \\
1.35182743+2.71567536 i \\
1.96236424-0.66176633 i\end{array}$ \\
\hline 8 & $\begin{array}{l}\text { vol }=1.41406104 \\
\mathrm{CS}=-0.11826873 \\
H_{1}=\mathbb{Z}_{10} \\
\text { Isom }=D_{2}\end{array}$ & $\begin{array}{l}(\times 1) \\
(\times 1) \\
(\times 2) \\
(\times 2)\end{array}$ & $\begin{array}{l}0.36489469-1.62824351 i * \\
0.84359872+1.40771544 i * \\
0.85802126-2.84667560 i \\
0.98118212+2.81070949 i\end{array}$ & $\begin{array}{l}(\times 1) \\
(\times 1) \\
(\times 1) \\
(\times 2)\end{array}$ & $\begin{array}{l}1.16920737-1.29244440 i \\
1.70794352+1.36945410 i \\
1.82127130-0.51794798 i \\
1.86598419+2.11045463 i\end{array}$ \\
\hline $5,-$ & $\begin{array}{l}\text { vol }=1.42361190 \\
\mathrm{CS}=-0.11256515 \\
H_{1}=\mathbb{Z}_{35} \\
\text { Isom }=D_{2}\end{array}$ & $\begin{array}{l}(\times 1) \\
(\times 2) \\
(\times 2) \\
(\times 1)\end{array}$ & $\begin{array}{l}0.35237160-1.75553066 i * \\
0.72219447-2.56160460 i \\
0.83247810+2.48715981 i \\
0.96132230+2.08327469 i *\end{array}$ & $\begin{array}{l}(\times 1) \\
(\times 2) \\
(\times 2) \\
(\times 2)\end{array}$ & $\begin{array}{l}1.22377342-2.11754199 i \\
1.74409578-2.80561863 i \\
1.75039321-2.25499321 i \\
1.86082909+1.79430243 i\end{array}$ \\
\hline 10 & $\begin{array}{l}\text { vol }=1.44069901 \\
\mathrm{CS}=-0.24006071 \\
H_{1}=\mathbb{Z}_{3} \\
\text { Isom }=D_{2}\end{array}$ & $\begin{array}{l}(\times 1) \\
(\times 1) \\
(\times 2) \\
(\times 1)\end{array}$ & $\begin{array}{l}0.36152158+3.05182035 i \\
0.79662309+1.47505982 i \\
1.25897223+1.99299289 i \\
1.26705358-1.32270789 i\end{array}$ & $\begin{array}{l}(\times 1) \\
(\times 2) \\
(\times 2)\end{array}$ & $\begin{array}{l}1.65279232+0.60455578 i \\
1.73929436+2.21381084 i \\
1.97217761-1.82481627 i\end{array}$ \\
\hline
\end{tabular}

TABLE 1. For each of the ten smallest known closed hyperbolic three-manifolds, all of which are orientable, we give: a surgery description that embodies the manifold's full symmetry group; the volume; the Chern-Simons invariant $\left(\bmod \frac{1}{2}\right)$; the first homology; the symmetry group; and the length spectrum to $\lambda=2.0$, with entries corresponding to core curves marked with asterisks. $D_{n}=$ dihedral group of order $2 n ; S_{16}=$ semidihedral group of order 16, with presentation $\left\langle x, y \mid x^{8}=y^{2}=1, y^{-1} x y=x^{3}\right\rangle$. 


\section{Remarks on Table 1}

1. E. Molnár [1992] computes the symmetry group of manifold \#1 as the group of outer automorphisms of its fundamental group. He finds it to be $\mathbb{Z}_{2} \times D_{3} \approx D_{6}$, in agreement with SnapPea's calculation.
2. All these manifolds, except for \#3, are chiral. For \#3, the surgery description displays only the orientation-preserving symmetries. For the orientation-reversing symmetries, do a full twist on the $(2,1)$ component and take the mirror image. See also [Gorenstein 1968, Thm. 4.4].

\begin{tabular}{|c|c|c|c|c|c|}
\hline $1=\operatorname{WLM}(3,1)$ & $\begin{array}{l}\text { vol }=2.02988321 \\
H_{1}=\mathbb{Z} \\
\text { Isom }=\mathbb{Z}_{2}\end{array}$ & $\begin{array}{l}(\times 1) \\
(\times 1) \\
(\times 3)\end{array}$ & $\begin{array}{l}0.48121183 \\
1.08707014+1.72276845 i * \\
1.44363548\end{array}$ & $\begin{array}{l}(\times 1) \\
(\times 4) \\
(\times 1)\end{array}$ & $\begin{array}{l}1.66288589+2.39212379 i \\
1.83178061+2.34978827 i \\
1.96497838+1.70869154 i\end{array}$ \\
\hline $2=\operatorname{WLM}(1,2)$ & $\begin{array}{l}\text { vol }=2.56897060 \\
H_{1}=\mathbb{Z} \\
\text { Isom }=\mathbb{Z}_{2}\end{array}$ & $\begin{array}{l}(\times 1) \\
(\times 1) \\
(\times 1) \\
(\times 2)\end{array}$ & $\begin{array}{l}0.65450441+3.04403773 i * \\
0.75552584 \\
1.01953372 \\
1.35092654+1.59574665 i\end{array}$ & $\begin{array}{l}(\times 2) \\
(\times 1) \\
(\times 2)\end{array}$ & $\begin{array}{l}1.57136773 \\
1.67747230+2.44874167 i \\
1.95872229\end{array}$ \\
\hline $3=\operatorname{WLM}(4,1)$ & $\begin{array}{l}\text { vol }=2.66674478 \\
H_{1}=\mathbb{Z}+\mathbb{Z}_{2} \\
\text { Isom }=\mathbb{Z}_{2}\end{array}$ & $\begin{array}{l}(\times 1) \\
(\times 1) \\
(\times 1) \\
(\times 2)\end{array}$ & $\begin{array}{l}0.63297432+1.36217964 i * \\
0.65847895 \\
1.14621583 \\
1.60863599\end{array}$ & $\begin{array}{l}(\times 2) \\
(\times 1) \\
(\times 1) \\
(\times 2)\end{array}$ & $\begin{array}{l}1.67235274+2.25870430 i \\
1.71593285+2.62768189 i \\
1.90806357+0.64608883 i \\
1.97543685\end{array}$ \\
\hline $4=\operatorname{WLM}(3,2)$ & $\begin{array}{l}\text { vol }=2.82812209 \\
H_{1}=\mathbb{Z} \\
\text { Isom }=\mathbb{Z}_{2}\end{array}$ & $\begin{array}{l}(\times 1) \\
(\times 1) \\
(\times 1) \\
(\times 2)\end{array}$ & $\begin{array}{l}0.56239915+2.81543089 i * \\
0.62155734 \\
1.20016235 \\
1.53918373+1.83957682 i\end{array}$ & $\begin{array}{l}(\times 1) \\
(\times 2) \\
(\times 2)\end{array}$ & $\begin{array}{l}1.75331810+2.91070443 i \\
1.76640534 \\
1.86467203\end{array}$ \\
\hline $5=\operatorname{WLM}(5,1)$ & $\begin{array}{l}\text { vol }=2.98912028 \\
H_{1}=\mathbb{Z} \\
\text { Isom }=\mathbb{Z}_{2}\end{array}$ & $\begin{array}{l}(\times 1) \\
(\times 1) \\
(\times 1)\end{array}$ & $\begin{array}{l}0.42347382+1.13005305 i * \\
0.74532073 \\
1.03193587\end{array}$ & $\begin{array}{l}(\times 2) \\
(\times 1) \\
(\times 2)\end{array}$ & $\begin{array}{l}1.62011660+2.19496331 i \\
1.73774325+2.76577738 i \\
1.76722808\end{array}$ \\
\hline $6=\operatorname{WLM}(1,3)$ & $\begin{array}{l}\text { vol }=3.14850983 \\
H_{1}=\mathbb{Z} \\
\text { Isom }=\mathbb{Z}_{2}\end{array}$ & $\begin{array}{l}(\times 1) \\
(\times 1) \\
(\times 1)\end{array}$ & $\begin{array}{l}0.31693581+2.05306079 i * \\
0.83274313 \\
0.93173621\end{array}$ & $\begin{array}{l}(\times 2) \\
(\times 1) \\
(\times 2)\end{array}$ & $\begin{array}{l}1.44513337+1.80715330 i \\
1.74233607+2.80199757 i \\
1.88440174\end{array}$ \\
\hline $7=\mathrm{WLM}(5,2)$ & $\begin{array}{l}\text { vol }=3.16396323 \\
H_{1}=Z \\
\text { Isom }=\mathbb{Z}_{2}\end{array}$ & $\begin{array}{l}(\times 1) \\
(\times 1) \\
(\times 1)\end{array}$ & $\begin{array}{l}0.33063552+2.74516418 i * \\
0.71540874 \\
1.06946994\end{array}$ & $\begin{array}{l}(\times 2) \\
(\times 1) \\
(\times 2)\end{array}$ & $\begin{array}{l}1.57378049+2.04060973 i \\
1.76113786+3.04618408 i \\
1.90350919\end{array}$ \\
\hline $8=\operatorname{WLM}(6,1)$ & $\begin{array}{l}\text { vol }=3.17729328 \\
H_{1}=\mathbb{Z}+\mathbb{Z}_{4} \\
\text { Isom }=\mathbb{Z}_{2}\end{array}$ & $\begin{array}{l}(\times 1) \\
(\times 1) \\
(\times 1)\end{array}$ & $\begin{array}{l}0.30468893+0.96535911 i * \\
0.79283563 \\
0.97582913\end{array}$ & $\begin{array}{l}(\times 2) \\
(\times 1) \\
(\times 2)\end{array}$ & $\begin{array}{l}1.59447927+2.15028807 i \\
1.74828597+2.85569824 i \\
1.86973590\end{array}$ \\
\hline $9=\operatorname{WLM}(2,3)$ & $\begin{array}{l}\text { vol }=3.17729328 \\
H_{1}=\mathbb{Z}+\mathbb{Z}_{4} \\
\text { Isom }=\mathbb{Z}_{2}\end{array}$ & $\begin{array}{l}(\times 1) \\
(\times 1) \\
(\times 1)\end{array}$ & $\begin{array}{l}0.30468893+2.17623355 i * \\
0.79283563 \\
0.97582913\end{array}$ & $\begin{array}{l}(\times 2) \\
(\times 1) \\
(\times 2)\end{array}$ & $\begin{array}{l}1.46449605+1.83536048 i \\
1.74828597+2.85569824 i \\
1.86973590\end{array}$ \\
\hline $10=\operatorname{WLM}(4,3)$ & $\begin{array}{l}\text { vol }=3.27587164 \\
H_{1}=\mathbb{Z}+\mathbb{Z}_{2} \\
\text { Isom }=\mathbb{Z}_{2}\end{array}$ & $\begin{array}{l}(\times 1) \\
(\times 1) \\
(\times 1)\end{array}$ & $\begin{array}{l}0.25226126+1.94569043 i * \\
0.76061802 \\
1.01341912\end{array}$ & $\begin{array}{l}(\times 2) \\
(\times 1) \\
(\times 2)\end{array}$ & $\begin{array}{l}1.51270014+1.92571859 i \\
1.76017564+3.02099168 i \\
1.92982197\end{array}$ \\
\hline
\end{tabular}

TABLE 2. Low-volume closed nonorientable hyperbolic three-manifolds, with same data as in Table 1 (minus the Chern-Simons invariant). For the notation WLM, see remarks on next page. 
3. All known surgery descriptions for manifold \#3 involve negatively oriented tetrahedra, so SnapPea cannot verify directly that the curves it drills out are isotopic to the geodesics in their respective homotopy classes. To make SnapPea's results rigorous, we computed a fundamental domain for this manifold and manually drilled out the unique geodesic of length $1.31 \ldots$ (the old-fashioned way, with pencil and paper) to check that its complement was the same as the one SnapPea had found. This is the only example in the tables for which SnapPea's algorithm failed. Among the 11,031 closed manifolds studied in [Hodgson and Weeks], SnapPea's algorithm failed for four additional examples, but in each case the correct symmetry group was found with human assistance.

\section{Remarks on Table 2}

1. Unlike the results in Table 1, which are backed by extensive and careful computer searches, the manifolds in this table should be considered only tentative candidates for the smallest closed nonorientable hyperbolic three-manifolds. All happen to be Dehn fillings on a manifold, denoted WLM, which is closely related to the Whitehead link complement. WLM is nonorientable but has an orientable cusp. The cusp shape is a $2: 1$ rectangle (that is, its conformal invariant is $2 i$ ); the Dehn fillings in the table are expressed relative to the basis (short side, long side). The length of the core curve is marked with an asterisk in the accompanying length spectrum.

2. The smallest known nonorientable closed hyperbolic three-manifold has more than twice the volume of the smallest orientable one. Cameron Gordon has pointed out that this may be because a nonorientable manifold has infinite homology, and therefore contains a two-sided nonseparating incompressible surface (compare [Hempel 1976, Lemmas 6.6 and 6.7]). The closed manifolds obtained by Dehn filling on WLM not only have the same volumes as the cusped manifolds obtained by Dehn filling on one cusp of the Whitehead link WL, but the complex lengths of the surgery core curves also coincide exactly. This is only one example of a much broader correspondence between cusped and closed manifolds that the authors are continuing to explore. Manifolds \#8 and \#9 have the same volume and first homology group as the cusped manifolds $\mathrm{WL}(4,1)$ and $\mathrm{WL}(4,-3)$, which are discussed at length in [Hodgson et al. 1992].

\section{ACKNOWLEDGMENTS}

Hodgson's work is partially supported by the Australian Research Council. Weeks thanks the Geometry Center (NSF Science and Technology Research Center for Computation and Visualization

\begin{tabular}{|c|c|c|c|c|}
\hline 1 & 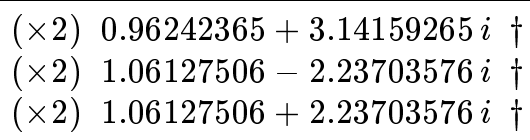 & 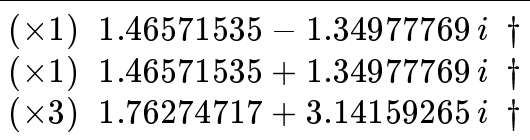 & $\begin{array}{l}(\times 2) \\
(\times 2)\end{array}$ & $\begin{array}{l}1.85226606-2.44271453 i \dagger \\
1.85226606+2.44271453 i \dagger\end{array}$ \\
\hline 2 & $\begin{array}{ll}(\times 3) & 2.17414029+2.83764841 i \\
(\times 6) & 2.38952643\end{array}$ & $\begin{array}{ll}(\times 4) & 2.58766388+1.75883426 i \\
(\times 3) & 2.63391579\end{array}$ & $\begin{array}{l}(\times 6) \\
(\times 12)\end{array}$ & $\begin{array}{l}2.92156323+1.38174409 i \\
2.99322285\end{array}$ \\
\hline 3 & 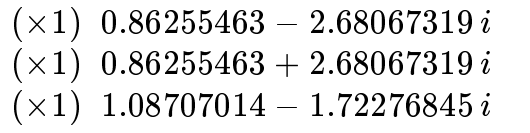 & $\begin{array}{ll}(\times 1) & 1.08707014+1.72276845 i \\
(\times 2) & 1.20805664-2.53724976 i \\
(\times 2) & 1.20805664+2.53724976 i\end{array}$ & $\begin{array}{l}(\times 1) \\
(\times 1) \\
(\times 1)\end{array}$ & $\begin{array}{l}1.66288589-2.39212379 i \dagger \\
1.66288589+2.39212379 i \dagger \\
1.76274717\end{array}$ \\
\hline
\end{tabular}

TABLE 3. Length spectra of sample hyperbolic three-orbifolds, as computed by SnapPea. Orbifold \#1 is $\mathbb{H}^{3} / \operatorname{PSL}(2, \mathbb{Z}[i])$, with generators computed by Pat Callahan. Orbifold \#2 is the mirrored regular ideal tetrahedron. Orbifold \#3 is obtained from the figure eight knot by $(4,0)$ surgery. The length spectrum for \#2 is shown to $\lambda=3.0$, the others to $\lambda=2.0$. The symbol $\dagger$ indicates that a geodesic is topologically a mirrored interval, not a circle; in this case the given length and torsion are those of the circular geodesic in the (local) twofold cover. 
of Geometric Structures) for its support, and the University of Melbourne for its hospitality during the preparation of this manuscript.

\section{REFERENCES}

[Beardon 1983] A. Beardon, The Geometry of Discrete Groups, Springer, New York, 1983.

[Conner and Raymond 1972] P. Conner and F. Raymond, "Manifolds with few periodic homeomorphisms", pp. 1-75 in Proc. Second Conf. Compact Transformation Groups, Amherst, 1971, vol. 2, Lecture Notes Math. 299, Springer, Berlin, 1972.

[Epstein and Penner 1988] D. B. A. Epstein and R. Penner, "Euclidean decompositions of noncompact hyperbolic manifolds", J. Diff. Geom. 27 (1988), 6780.

[Gorenstein 1968] D. Gorenstein, Finite Groups, Harper and Row, New York, 1968. Reprinted by Chelsea, New York, 1980.

[Hempel 1976] J. Hempel, 3-Manifolds, Princeton Univ. Press, Princeton, 1976.

[Henry and Weeks 1992] S. Henry and J. Weeks, "Symmetry groups of hyperbolic knots and links", J. Knot Theory and its Ramifications 1 (1992), 185201.

[Hildebrand and Weeks 1989] M. Hildebrand and J. Weeks, "A computer generated census of cusped hyperbolic 3-manifolds", pp. 53-59 in Computers and Mathematics (edited by E. Kaltofen and S. Watt), Springer, New York, 1989.

[Hodgson et al. 1992] C. Hodgson, R. Meyerhoff and J. Weeks, "Surgeries on the Whitehead link yield geometrically similar manifolds" pp. 195-206 in Topology '90 (edited by B. Apanasov et al.), de Gruyter, Berlin, 1992.
[Hodgson and Weeks] C. Hodgson and J. Weeks, "A census of closed hyperbolic three-manifolds", in preparation.

[Kojima 1988] S. Kojima, "Isometry transformations of hyperbolic three-manifolds", Topology Appl. 29 (1988), 297-307.

[Molnár 1992] E. Molnár, "On isometries of space forms", pp. 509-534 in Differential Geometry and Its Applications (Eger, 1989), North-Holland, Amsterdam, 1992.

[Sakai 1991] T. Sakai, "Geodesic knots in a hyperbolic 3-manifold", Kobe J. Math. 8 (1991), 81-87.

[Sakuma and Weeks 1995] M. Sakuma and J. Weeks, "The generalized tilt formula", Geometriae Dedicata 50 (1995), 1-9.

[Thurston 1979] W. Thurston, The Geometry and Topology of Three-Manifolds, lecture notes, Princeton University, 1979.

[Weeks 1993] J. Weeks, "Convex hulls and isometries of cusped hyperbolic 3-manifolds", Topology Appl. 52 (1993), 127-149.

[Weeks 1995] J. Weeks, SnapPea documentation. The program and accompanying documentation are available free of charge by anonymous $\mathrm{ftp}$ from geom.umn. edu in directory pub/software/snappea, or on a Macintosh diskette from Weeks.

\section{ELECTRONIC AVAILABILITY}

The authors have made a census of closed hyperbolic three-manifolds that were obtained by Dehn filling on a census of cusped hyperbolic three-manifolds [Hildebrand and Weeks 1989]. A full report on this census, which totals 11,031 distinct manifolds, will appear in [Hodgson and Weeks]. The symmetry groups of these manifolds are available for anonymous ftp from geom. umn. edu in directory pub/software/snappea/tables, along with their volumes, Chern-Simons invariants, homology groups and lengths of shortest geodesics. See also the bibliography entry [Weeks 1995] above. 
Craig D. Hodgson, Department of Mathematics, University of Melbourne, Parkville, Victoria 3052, Australia (cdh@mundoe.maths.mu.oz.au)

Jeffrey R. Weeks, The Geometry Center, University of Minnesota, 1300 South Second Street (suite 500), Minneapolis, MN 55454, USA (weeks@geom.umn.edu)

Received March 7, 1994; accepted in revised form February 1, 1995 